\title{
Convergence Behaviour of Iteratively Decoded Short Block-Codes in H.264 Joint Source and Channel Decoding
}

\author{
Nasruminallah and L. Hanzo \\ School of ECS, University of Southampton, SO17 1BJ, UK. \\ http: / / www-mobile.ecs. soton.ac.uk, \\ Email: $\{1 \mathrm{~h}\} @ e c s . s o t o n . a c . u k$
}

\begin{abstract}
We propose a novel class of Short Block Codes (SBCs) designed for guaranteed convergence to an infinitesimally low Bit Error Ratio (BER), which relies on the joint optimisation of soft-bit assisted iterative source and channel decoding. An iterative detection aided combination of SBC assisted Soft-Bit Source Decoding (SBSD) and a rate-1 precoder was used to evaluate the attainable performance of the proposed scheme for transmission of Data-Partitioned (DP) H.264 source coded video over correlated narrowband Rayleigh fading channels. Additionally, we demonstrated the effects of different SBCs having diverse minimum Hamming distances $\left[d_{H, \text { min }}\right]$ but identical coding rates on both the overall BER performance as well as on the objective video quality expressed in terms of the Peak Signal-to-Noise Ratio (PSNR). The convergence behaviour of the iterative decoding scheme using SBCs as a function of $d_{H, \text { min }}$ is analysed by utilising extrinsic information transfer (EXIT) charts. Explicitly, our experimental results show that the proposed error protection scheme using SBCs associated with $d_{H, \min }=6$ outperforms the identical-code-rate SBCs having $d_{H, \min }=3$ by about $3 \mathrm{~dB}$ at the PSNR degradation point of $1 \mathrm{~dB}$. Additionally, an $E_{b} / N_{0}$ gain of $27 \mathrm{~dB}$ is attained using iterative soft-bit source and channel decoding with the aid of rate- $\frac{1}{3}$ SBCs relative to the identical-rate benchmarker.
\end{abstract}

I. MOTIVATION AND BACKGROUND

There has been an increasing interest in multimedia transmission over wireless channels [1,2]. However, in wireless systems the data rate is limited by both the available bandwidth and transmission power. Various data compression standards have been developed to transmit multimedia signals over band-limited channels [3,4]. However, the removal of redundancy renders the compressed data stream more vulnerable to transmission errors. Therefore, simultaneously maintaining a high compression efficiency as well as a high integrity, when communicating over heterogeneous communication networks is of paramount importance [3]. As a result, the joint optimisation of traditionally separate functions leads to joint source and channel decoding (JSCD) in order to achieve the best possible performance in realistic practical scenarios, which has attracted considerable research interest [5-9]. The family of JSCD schemes involving the exploitation of the residual redundancy in the source-coded bit-stream as a form of error protection is of prime concern $[10,11]$. More specifically, an attractive SBSD scheme was proposed by Fingscheidt and Vary [10], in order to exploit the residual redundancy of the source-coded bit-stream for the convergence improvement of Iterative Source-Channel Decoding (ISCD) [11]. However, when using sophisticated state-of-the-art coding schemes, only modest residual redundancy remains in the source coded bitstream, therefore we propose to deliberately impose further redundancy on the source coded bit-stream with the aid of the novel class of SBCs proposed. In our investigations we used the H.264/AVC [12] source codec, which employs predictive coding and Variable Length Coding (VLC) techniques to achieve a high compression efficiency, hence rendering the compressed bit-stream vulnerable to transmission errors [3]. A single bit error in the coded stream may lead to the corruption of numerous future codewords. Furthermore, due to predictive coding the effects of channel errors may affect the neighboring video blocks owing to error propagation. Therefore the transmission of compressed video over wireless systems presents a challenging task. Error resilient Data Partitioning (DP) [12] has been incorporated in the H.264/AVC codec in order to mitigate the effects of channel errors. In the H.264/AVC codec DP results in three different types of streams, each containing specific sets of coding parameters having different level of importance. Various error resilient schemes have been proposed in [3], in order to alleviate these problems, but the price paid is a potentially reduced compression efficiency and increased computational complexity. Similarly, an iterative joint sourcechannel decoding procedure inspired by the concept of serial concatenated codes was presented in [6]. Moreover, a symbolbased soft-input a posteriori probability (APP) decoder was presented in [8], where the residual redundancy inherent in the compressed bit stream was exploited for improved error protection. On the other hand, a novel irregular variable length coding (IrVLC) scheme designed for near-capacity joint source and channel coding was advocated in [5]. Likewise, [13] advocates state-of-the-art system design principles and the performance of burst-by-burst adaptive transceivers for interactive cellular and cordless video telephony. Furthermore, a joint source-channel decoding method based on the MAP algorithm was proposed in [9].

Against this background, in this paper we propose novel $S B C s$ designed for guaranteed convergence of the iterative SBSD scheme advocated, leading to a $27 \mathrm{~dB}$ transmit-power gain at the Peak Signal-to-Noise Ratio (PSNR) degradation point of $1 \mathrm{~dB}$.

The rest of the paper is organised as follows. An overview of our system model is provided in Section II. Section III portrays our SBC based ISCD scheme accompanied by a design example, while its EXIT chart analysis is provided in Section IV. The performance of the proposed system is characterised with the aid of our simulation results in Section V. Finally we provide our conclusions in Section VI. 


\section{Iterative Source-ChanNel DeCODING} A. System Overview

The structure of our proposed ISCD videophone arrangement used as our design example for quantifying the performance of various SBC schemes is shown in Figure 1. At the transmitter side the H.264 video codec is used to compress the source video sequence and the generated bit-stream $x_{k}$ is mapped or encoded into the bit-string $x_{m}^{\prime}$ by employing the SBC scheme. Subsequently, the encoded bit-string $x_{m}^{\prime}$ is interleaved using the bit-interleaver $\Pi$ of Figure 1, yielding the interleaved sequence $\bar{x}_{m}$, which is encoded by the rate- 1 precoder of Table II before transmission.

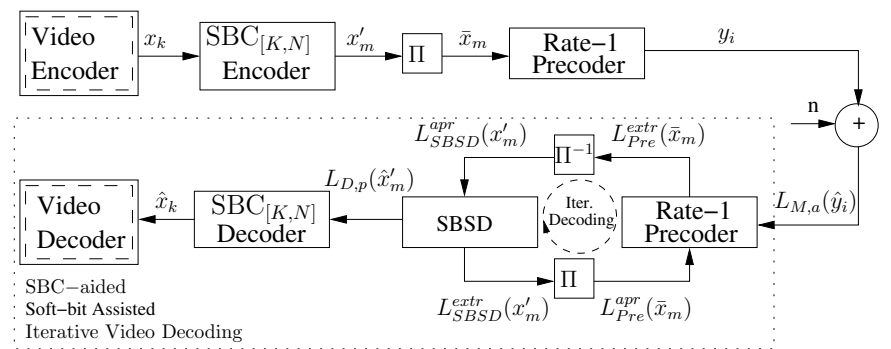

Fig. 1. The Proposed Iterative Source-Channel Decoding System Model.

In the iterative decoder of Figure 1 , the extent of the statistical independence of the extrinsic information provided by an interleaver is always related to its length [7]. Therefore instead of performing the ISCD operation on the various frame slices independently, we concatenated all the bits generated by the MBs of the slices within a given frame, which results in a longer interleaver and hence improves the attainable performance of iterative decoding without extending the video delay. The precoded bit-stream $y_{i}$ is QPSK modulated and transmitted over a temporally correlated narrowband Rayleigh fading channel, associated with the normalised Doppler frequency of $f_{d}=f_{D} T_{s}=0.01$, where $f_{D}$ is the Doppler frequency and $T_{s}$ is the symbol duration. At the receiver the soft-information obtained after QPSK demodulation is forwarded to the inner decoder. The extracted extrinsic information is then exchanged between the inner and outer decoder of Figure 1, in order to attain the lowest possible BER [11].

\section{B. The Soft-bit Source Decoding}

The details of computing the extrinsic Log-LikelihoodRatios (LLRs) using SBSD for the zero-order Markov model can be obtained from [11]. More specifically, according to our coding procedure the source-encoded bit-stream is partitioned into $M=2^{K}$-ary, or $K$-bit symbols, and will be termed as the information word to be encoded by the proposed SBC, each with a different probability of occurance. The redundancy of the source bit-stream is then characterised with the aid of the non-uniform $M=2^{K}$-ary symbol probability distribution $P\left[S_{K}(\tau)\right]$, where $S_{K}(\tau)=\left[S_{K}(1), S_{K}(2), \cdots S_{K}(M)\right]$, with $K$ denoting the number of bits in each $M=2^{K}$-ary symbol $^{1}$. Provided that the bits of an $M=2^{K}$-ary symbol may be considered independent of each other, the channels'

\footnotetext{
${ }^{1}$ The choice of how many bits are specifically grouped into an $M=2^{K}$ ary symbol represents an important system parameter, but due to space limitations this issue is not detailed in this paper.
}

output information generated for the $\tau$-th $K$-bit symbol is provided by the product of each of the constituent single-bit probabilities given as:

$$
P\left[\hat{y}_{\tau} \mid y_{\tau}\right]=\prod_{k=1}^{K} P\left[\hat{y}_{\tau}(k) \mid y_{\tau}(k)\right],
$$

where $\hat{y}_{\tau}$ and $y_{\tau}$ are the corresponding transmitted and received $K$-bit source sequences respectively. For each desired bit $\left[y_{\tau}(\lambda)\right]$, the extrinsic channel output information $P\left[\hat{y}_{\tau}^{[e x t]} \mid y_{\tau}^{[e x t]}\right]$ is expressed as:

$$
P\left[\hat{y}_{\tau}^{[e x t]} \mid y_{\tau}^{[e x t]}\right]=\prod_{k=1, k \neq \lambda}^{K} P\left[\hat{y}_{\tau}(k) \mid y_{\tau}(k)\right] .
$$

Finally, the resultant extrinsic LLR value can be acquired for each bit of the $\tau$-th symbol by combining its channel output information and the a-priori knowledge of the corresponding $\tau$-th symbol as $[10,11]$ :

$$
\log \left(\frac{\sum_{y_{\tau}^{[e x t]}} P\left[y_{\tau}^{[e x t]} \mid y_{\tau}(\lambda)=+1\right] \cdot P\left[\hat{y}_{\tau}^{[e x t]} \mid y_{\tau}^{[e x t]}\right]}{\sum_{y_{\tau}^{[e x t]}} P\left[y_{\tau}^{[e x t]} \mid y_{\tau}(\lambda)=-1\right] \cdot P\left[\hat{y}_{\tau}^{[e x t]} \mid y_{\tau}^{[e x t]}\right]}\right) .
$$

The trait of our proposed SBC coding technique is that its generic and hence it is applicable to arbitrary speech, audio and video source codecs. However in our design example we characterised the source coded bit-stream's redundancy with the aid of the non-uniform $M$-ary symbol probability distribution ${ }^{2}$ using the H.264/AVC video encoded bit-stream of the 300-frame "Akiyo" video sequence, the 150-frame "MissAmerica" video clip and the 300-frame "Mother\&Daughter" video sequence, which were used as training sequences.

\section{Short Block Code BASEd Iterative SOURCE CHANNEL DECODING}

\section{A. The Criterion for Iterative Convergence}

The purpose of ISCD is to allow the constituent inner and outer decoders in order to assist each other in an iterative fashion to glean the highest possible extrinsic information from each other. In this senario the achievable performance of SBSD is limited by the factor that its achievable iteration gain is dependent on the residual redundancy or correlation that remains in the coded bit pattern $x_{i}$. Therefore, despite using limited-delay, limited-complexity, lossy compression, which typically results in residual redundancy in the compressed stream, the achievable performance improvements of SBSD may remain limited. To elaborate a little further, the lower the video bitrate, the less extrinsic information may be generated from the source-coded stream. It may be observed from the simulation results of [14] that using SBSD typically results in negligible system performance improvements beyond two decoding iterations. Hence, in order to improve the achievable ISCD performance gain, we artificially introduce redundancy

\footnotetext{
${ }^{2}$ The resultant $M$-ary source word probability distribution for the $\mathrm{SBC}_{[K, N]}$ coding schemes used in our design example is not provided in this paper owing to space limitations.
} 
in the source coded bit-stream using a carefully designed technique, which we refer to as an SBC scheme. The novel philosophy of our SBC design is based on exploiting a specific property of EXIT charts. More explicitly, an iterative decoding aided receiver is capable of near-capacity operation at an infinitesimally low BER, if there is an open tunnel between the EXIT curves of the inner and outer constituent component decoders. The sufficient and necessary condition for this iterative detection convergence criterion to be met was shown by Kliewer et al [15] to be that the legitimate codewords should have $d_{H, \text { min }}=2$. With $d_{H, \text { min }}=2$ the ISCD scheme becomes capable of achieving the highest possible source entropy denoted as $H(X)=L_{S B S D}^{\text {extr }}=1 \mathrm{bit}$, provided that the input a-priori information of the SBSD is perfect, i.e. we have $H(X)=L_{S B S D}^{a p r i}=1$ bit. This motivates the design of our novel SBC coding scheme, because it is plausible that using our design procedure all legitimate SBC codewords having a specific mapping-rate equivalent to the reciprocal of the classic code-rate results in a code-table satisfying the condition of $d_{H, \min }=2$.

\section{B. Algorithm of Short Block Codes}

The fundamental principle of our $\operatorname{SBC}_{[K, N]}$ encoding algorithm is based on the necessary and sufficient condition outlined in Section III-A for achieving perfect iterative convergence to an infintisimally low BER. The proposed $\mathrm{SBC}_{[K, N]}$ encoding algorithm partitions the video-stream $x_{k}$ into $K$-bit source symbols, which will be encoded into $N=(K+P)$ bits, where $P$ represents the number of redundant bits per $K$-bit information word.

For $P=(m \times K)$ with $m \geq 1$, we propose the corresponding $\mathrm{SBC}_{[K, N]}$-encoding procedure, which results in a gradual increase of $d_{H, \text { min }}$ for the coded symbols upon increasing both $K$ and $N$ of the $\operatorname{SBC}_{[K, N]}$, while the coderate is fixed. Using this $K$ to $N$-bit encoding method, first $I=[(m-1) \times K]$ number of redundant bits $r_{\tau}(i)$, for $i=1,2 \cdots I$ are concatenated to the $\tau$-th $K$-bit source symbol by repeated concatenation of $K$ additional source coded bits $(m-1)$ times, yielding a total of $[(m-1) \times K]$ bits. Finally, the last set of $K$ redundant bits $r_{\tau}(k)$, for $k=1,2 \cdots K$ is generated by calculating the exclusive $O R(X O R)$ function of the $K$ source bits $b_{\tau}(j)$, while setting $b_{\tau}[j=k]$ equal to 0 , yielding:

$$
\begin{array}{r}
r_{\tau}(k)=\left[b_{\tau}(1) \oplus b_{\tau}(2) \ldots \oplus b_{\tau}(K)\right] ; \text { for } k=1,2 \cdots K, \\
\text { while setting } b_{\tau}(k)=0,
\end{array}
$$

where $\oplus$ represents the $X O R$ operation.

Using this method a carefully controlled redundancy is imposed by the specific rate $r=\left[\frac{K}{N}\right] \mathrm{SBC}_{[K, N]}$ to ensure that the resultant $N$-bit codewords exhibit a $d_{H, \text { min }} \geq 2$ between the $M=2^{K}$ number of legitimate $K$-bit source code words.

\section{Design Example}

Let us now demonstrate the power of SBCs with the aid of a design example. As an example, the $\operatorname{SBC}_{[K, N]}$-encoded symbols generated by applying this algorithm for the different rate- $\frac{1}{3}$ SBC schemes along with their corresponding $d_{H, \text { min }}$ is summarised in Table I for the specific case of incorporating the redundant bits $r_{\tau}(k)$, for $k=1,2 \cdots K$ at the right of the $\tau$-th $K$-bit source symbol. As evident from Table I, only $2^{K}$ out of the $2^{N}$ possible $N$-bit symbols are legitimate in the mapped source coded bit-stream, which exhibits a non-uniform probability of occurance for the $N$-bit source symbols. The TABLE I

DIFFERENT SBCS WITH CORRESPONDING SYMBOLS AND $d_{H, \text { min }}$

\begin{tabular}{|l|l|c|}
\hline SBC Type & Symbols in Decimal & $d_{H, \text { min }}$ \\
\hline \hline Rate-1 SBC & $\{0,1\}$ & 1 \\
\hline Rate- $\frac{1}{3} \mathrm{SBC}_{[2,6]}$ & $\{0,22,41,63\}$ & 3 \\
\hline Rate- $\frac{1}{3} \mathrm{SBC}_{[3,9]}$ & $\{0,78,149,219,291,365,438,504\}$ & 4 \\
\hline Rate- $\frac{1}{3} \mathrm{SBC}_{[4,12]}$ & $\{0,286,557,819,1099,1365,1638,1912,2183$, & 5 \\
& $2457,2730,2996,3276,3538,3809,4095\}$ & 6 \\
\hline Rate- $\frac{1}{3} \mathrm{SBC}_{[5,15]}$ & $\{0,1086,2141,3171,4251,5285,6342,7416$, & \\
& $8471,9513,10570,11636,12684,13746,14801$, & \\
& $15855,16911,17969,19026,20076,21140,22186$, & \\
& $23241,24311,25368,26406,27461,28539,29571$, & \\
& $30653,31710,32736\}$ & \\
\hline
\end{tabular}

coding parameters of the different SBC schemes used in our design example are shown in Table II. Observe from the table that an overall code-rate of $R=\frac{1}{3}$ was maintained by using a concatenated rate- 1 precoder in order to accommodate the different rate- $\frac{1}{3}$ SBCs of Table I. Moreover, for the rate-1 SBC benchmarker scheme the rate- $\frac{1}{3}$ concatenated RSC is used as inner code in order to achieve an iteration gain, while keeping the overall bit-rate budget constant.

TABLE II

CODE RATES FOR DIFFERENT ERROR PROTECTION SCHEMES

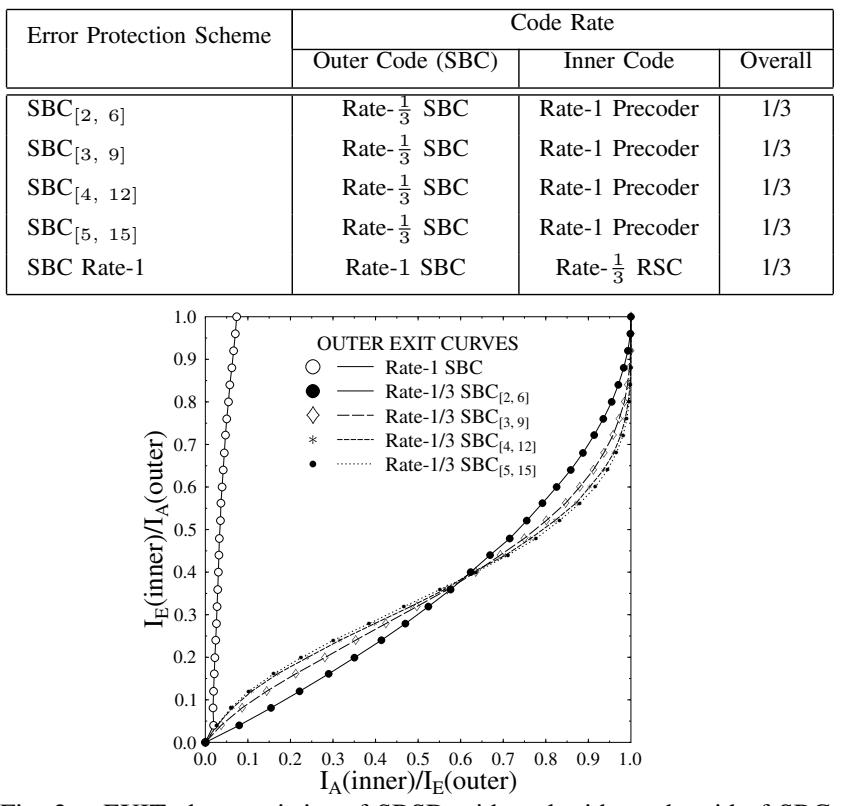

Fig. 2. EXIT characteristics of SBSD with and without the aid of SBCs.

IV. EXIT CHART ANALYSIS

The EXIT characteristics of the SBSD scheme of Figure 1 using either the rate- $1^{3}$ or the rate- $\frac{1}{3} \operatorname{SBC}_{[K, N]}$ schemes shown in Table I are portrayed in Figure 2. More specifically, the EXIT curve of SBSD using rate- $\frac{1}{3}$ SBCs does indeed reach to the top right corner of the EXIT chart at $\left(I_{A}, I_{E}\right)=(1,1)$ and hence results in an infinitesimally low BER. By contrast,

\footnotetext{
${ }^{3}$ For the sake of using a unified terminology, we refer to the scheme using no SBC as the rate- $1 \mathrm{SBC}$.
} 


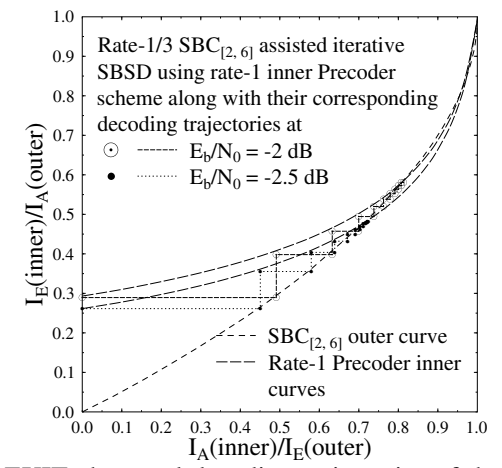

Fig. 3. The EXIT chart and decoding trajectories of the $\mathrm{SBC}_{[2,6]}$ scheme.

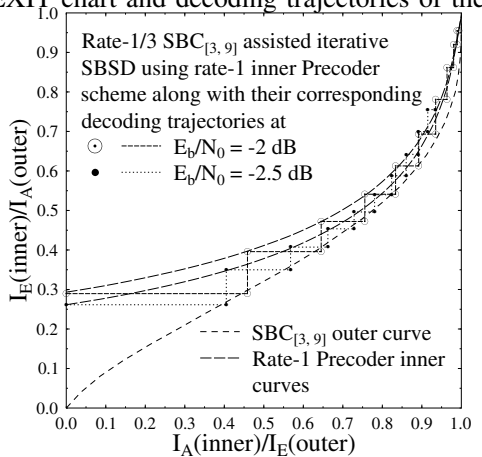

Fig. 4. The EXIT chart and decoding trajectories of the $\left.\mathrm{SBC}_{[3}, 9\right]$ scheme. the SBSD scheme using a rate-1 SBC, i.e. no SBC fails to do so. The actual decoding trajectories of the various error protection schemes employing the different rate- $\frac{1}{3}$ outer SBCs as well as using the rate- 1 inner precoder detailed in Table II were recorded both at $E_{b} / N_{0}=-2 d B$ and $-2.5 d B$, as portrayed in Figures 3, 4, 5, and 6, respectively. These trajectories were recorded by acquiring the mutual information at the input and output of both the inner and outer decoder during the bit-by-bit Monte-Carlo simulation of the iterative soft-bit source and channel decoding algorithm. It may be analysed from the EXIT trajectories of Figures 3, 4, 5, and 6 that as expected, the convergence behaviour of the SBCs improves upon increasing $d_{H, \text { min }}$.

\section{System Performance Results}

In this section we present our performance results characterising the proposed system. A 45-frame "Akiyo" video sequence [3] represented in (176x144)-pixel Quarter Common Intermediate Format (QCIF) and encoded using the H.264/AVC JM 13.2 reference video codec at 15 frames-persecond ( $f p s)$ at the target bitrate of $64 \mathrm{kbps}$ was used as our test sequence. Each QCIF frame was partitioned into 9 slices and each slice was composed of $11 \mathrm{MBs}$. In order to reduce error propagation each intra-coded 'I' frame is followed by 44 predicted ' $\mathrm{P}$ ' frames, corresponding to 3 seconds lag between two consecutive 'I' frames at $15 \mathrm{fps}$. Additionally, we incorporated error resilience features, such as DP and intra-frame coded MB updates of three randomly dispersed MBs per frame, to control the effects of error propagation. The insertion of ' $\mathrm{B}$ ' pictures results in an unacceptable loss of lip-synchronisation as a result of the corresponding delay incurred due to the bi-directionally predicted video coding operations [12], and hence was avoided. The remaining system parameters of our experimental setup are listed in Table III.

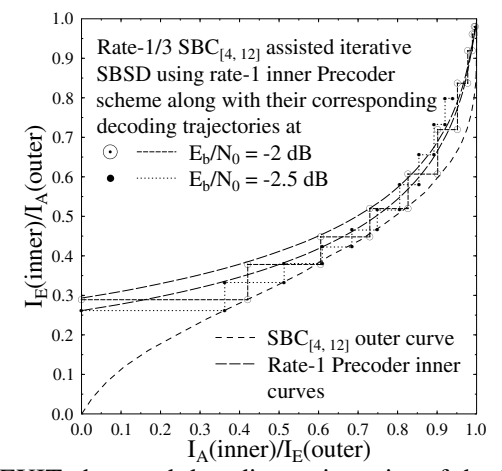

Fig. 5. The EXIT chart and decoding trajectories of the $\mathrm{SBC}_{[4,12]}$ scheme.

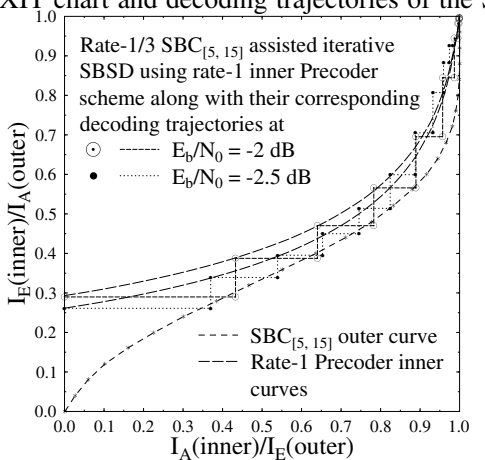

Fig. 6. The EXIT chart and decoding trajectories of the $\left.\mathrm{SBC}_{[5}, 15\right]$ scheme. Keeping in view the videophone senario, error resilient enTABLE III

SYSTEMS PARAMETERS

\begin{tabular}{|l|l|l|l|}
\hline System Parameters & Value & System Parameters & Value \\
\hline \hline Source Coding & H.264/AVC & No of MB's/Slice & 11 \\
Bit Rate (Kbps) & 64 & Intra-frame MB & 3 \\
Frame Rate (fps) & 15 & update/frame & \\
No of Slices/frame & 9 & & \\
\hline Inner Code & Rate-1 Precoder & Over-all Code Rate & $1 / 3$ \\
\hline Modulation Scheme & QPSK & Channel & $\begin{array}{l}\text { Correlated } \\
\text { Rayleigh } \\
\text { Number of }\end{array}$ \\
Transmitters, $N_{t}$ & 1 & & Fading \\
Number of & 1 & Normalised & \\
Receivers, $N_{r}$ & & $\begin{array}{l}\text { Doppler } \\
\text { Frequency }\end{array}$ & 0.01 \\
Interleaver Length & $\approx(64000 / 15)$ & Frequen \\
\hline
\end{tabular}

coding techniques, such as Flexible Macro-block Ordering (FMO) [12] and the employment of multiple reference frames for inter-frame motion compensation were turned off, because despite their substantially increased complexity they typically result in modest video performance improvements in low-motion head-and-shoulders video sequences, such as the "Akiyo" clip. Additionally, only the immediately preceding frame was used for motion search, which results in a reduced computational complexity compared to using multiple reference frames. Moreover, due to the limited residual redundancy inherent in the source encoded bit-stream and for the sake of reducing the computational complexity imposed, we limited the number of iterations between the RSC and SBSD decoders to $I_{t}=5$, when using a rate- $1 \mathrm{SBC}-$ i.e. no SBC. By contrast we used $I_{t}=10$ iterations, when applying SBCs having a rate below unity. For the sake of increasing the confidence in our results, we repeated each 45-frame experiment 160 times and averaged the generated results. Figure 7 presents the performance of the various rate- $\frac{1}{3} \mathrm{SBC}$ based error protection schemes of Table I in terms of the attainable BER, while their comparison with the rate- 1 SBC based schemes is offered 
in Figures 9. Finally, the performance trends expressed in terms of the $P S N R$ versus $E_{b} / N_{0}$ curves are portrayed in Figures 8 and 10. It may be observed in Figure 8 that the $\mathrm{SBC}_{[5,15]}$ scheme having $d_{H, \text { min }}=6$ provides the best $P S N R$ performance among the four different SBC schemes of Table II across the entire $E_{b} / N_{0}$ region considered. Its also observed in Figure 10 that upon using SBSD in conjunction with the rate- 1 outer SBC and rate- $\frac{1}{3}$ inner RSC results in a worse $P S N R$ performance than the employment of rate$\frac{1}{3}$ outer SBCs combined with the rate- 1 inner precoder, as mentioned in Table II, although they have the same over-all code rate. Quantitatively, using the rate- $\frac{1}{3}$ SBCs of Table II, an additional $E_{b} / N_{0}$ gain of upto $27 \mathrm{~dB}$ may be achieved over the identical-rate benchmarker scheme dispensing with SBC.

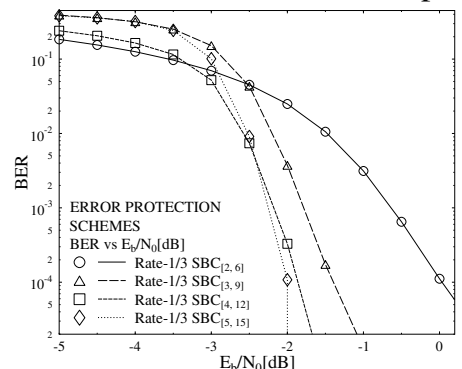

Fig. 7. BER performance of the various error protection schemes.

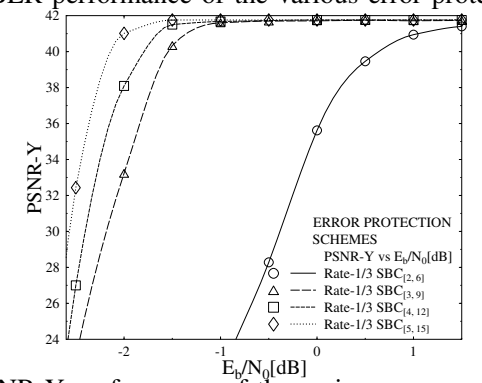

Fig. 8. PSNR-Y performance of the various error protection schemes.

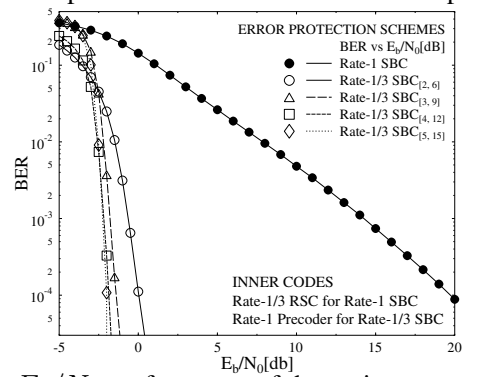

Fig. 9. BER vs $E_{b} / N_{0}$ performance of the various error protection schemes.

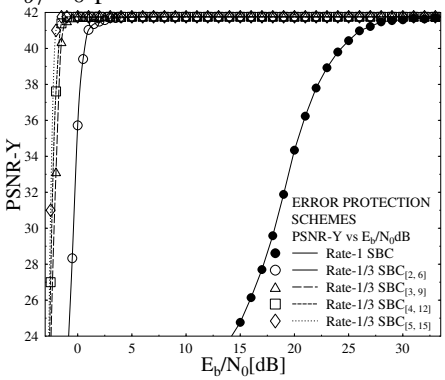

Fig. 10. PSNR-Y performance of various error protection schemes. VI. CONCLUSIONS

In this paper a generic low-complexity SBC coding scheme is proposed which satisfies the necessary and sufficient condition of $d_{H, \min }=2$ for iterative detection convergence for arbitrary SBSD-aided multimedia source codecs. We applied different error protection schemes considering the transmission of DP aided H.264/AVC coded video using carefully selected SBCs with diverse $d_{H, \text { min }}$. It was demonstrated that the bit-error correction capability of the ISCD scheme was significantly improved with the advent of rate- $\frac{1}{3}$ SBC scheme owing to the deliberate increase in redundancy of the source coded bit-stream. Additionally, the convergence behaviour of different SBC schemes in the ISCD paradigm was analysed using EXIT charts. The H.264/SBC/rate-1 precoder design example exhibited an $E_{b} / N_{0}$ gain of $3 \mathrm{~dB}$ at the $P S N R$ degradation point of $1 \mathrm{~dB}$, when using SBCs having $d_{H, \text { min }}=$ 6 compared to the identical-rate SBCs having $d_{H, \text { min }}=3$. Moreover, an $E_{b} / N_{0}$ gain of $27 \mathrm{~dB}$ was attained using iterative soft-bit source and channel decoding with the aid of rate- $\frac{1}{3}$ SBCs relative to the identical-rate benchmarker.

\section{REFERENCES}

[1] J. Streit and L. Hanzo, "Dual-mode vector-quantized low-rate cordless videophone systems forindoors and outdoors applications," IEEE Transactions on Vehicular Technology, vol. 46, pp. 340-357, May 1997.

[2] L. Hanzo, P. Cherriman, and E. L. Kuan, "Interactive cellular and cordless video telephony: State-of-the-art system design principles and expected performance," Proceedings of the IEEE, vol. 88, pp. 13881413, Sept. 2000.

[3] L. Hanzo, P. Cherriman, and J. Streit, Video Compression and Communications: From Basics to H.261, H.263, H.264, MPEG2, MPEG4 for DVB and HSDPA-Style Adaptive Turbo-Transceivers. Wiley-IEEE Press, 2007.

[4] L. Hanzo, C. Somerville, and J. Woodard, Voice and Audio Compression for Wireless Communications, 2nd Edition. Wiley-IEEE Press, 2007.

[5] R. G. Maunder, J. Wang, S. X. Ng, L. L. Yang, and L. Hanzo, "On the performance and complexity of irregular variable length codes for near-capacity joint source and channel coding," IEEE Transactions on Wireless Communications, vol. 7, pp. 1338-1347, Apr. 2008.

[6] A. Guyader, E. Fabre, C. Guillemot, and M. Robert, "Joint sourcechannel turbo decoding of entropy-coded sources," IEEE Journal on Selected Areas in Communications, vol. 19, pp. 1680-1696, Sept. 2001.

[7] R. G. Maunder, J. Kliewer, S. X. Ng, J. Wang, L. L. Yang, and L. Hanzo, "Joint iterative decoding of trellis-based VQ and TCM," IEEE Transactions on Wireless Communications, vol. 6, pp. 1327-1336, Apr. 2007.

[8] J. Kliewer and R. Thobaben, "Iterative joint source-channel decoding of variable-length codes using residual source redundancy," IEEE Transactions on Wireless Communications, vol. 4, pp. 919-929, May 2005.

[9] Y. Wang and S. Yu, "Joint source-channel decoding for H.264 coded video stream," IEEE Transactions on Consumer Electronics, vol. 51, pp. 1273-1276, Nov. 2005.

[10] T. Fingscheidt and P. Vary, "Softbit speech decoding: a new approach to error concealment," IEEE Transactions on Speech and Audio Processing, vol. 9, pp. 240-251, Mar. 2001.

[11] M. Adrat and P. Vary, "Iterative source-channel decoding: improved system design using exit charts," EURASIP J. Appl. Signal Process., vol. 2005, no. 1, pp. 928-941, 2005.

[12] J. Ostermann, J. Bormans, P. List, D. Marpe, M. Narroschke, F. Pereira, T. Stockhammer, and T. Wedi, "Video coding with H.264/AVC: tools, performance, and complexity," IEEE Circuits and Systems Magazine, vol. 4, no. 1, pp. 7-28, 2004.

[13] L. Hanzo, P. Cherriman, and E. L. Kuan, "Interactive cellular and cordless video telephony: State-of-the-art system design principles and expected performance," Proceedings of the IEEE, vol. 88, pp. 13881413, Sept. 2000.

[14] T. Hindelang, M. Adrat, T. Fingscheidt, and S. Heinen, "Joint source and channel coding: from the beginning until the 'EXIT'," European Transactions on Telecommunications, vol. 18, no. 8, pp. 851-858, 2007.

[15] J. Kliewer, N. Goertz, and A. Mertins, "Iterative source-channel decoding with markov random field source models," IEEE Transactions on Signal Processing, vol. 54, no. 10, pp. 3688-3701, Oct. 2006. 